\title{
¿En qué aspectos es más deficiente la alfabetización científica del alumnado de Bachillerato?
}

\author{
Mireia Balastegui \\ IES Jose Rodrigo Botet (Manises). España.balastegui_min@gva.es \\ Rafael Palomar iD \\ Departamento de Didáctica de la Ciencias Experimentales y Sociales. Universitat de València (España). \\ rafael.palomar@m.es \\ Jordi Solbes iD \\ Departamento de Didáctica de la Ciencias Experimentales y Sociales. Universitat de València (España). \\ jordi.solbes@uv.es
}

[Recibido: 9 diciembre 2019. Revisado: 27 mayo 2020. Aceptado: 25 junio 2020]

\begin{abstract}
Resumen: En el presente artículo se realiza un estudio sobre diversas definiciones de alfabetización científica. Basándonos en ellas se elabora una serie de indicadores de la misma y se presenta un diseño experimental que pretende evaluar el grado de alfabetización científica del alumnado del bachillerato científico tecnológico (GCT) y el de humanidades y ciencias sociales (GHS) en dichos aspectos, comparándolos entre sí. Los resultados ponen de manifiesto que conceptualmente el alumnado del GCT tiene un mayor grado de conocimiento científico que el alumnado GHS. En cambio, llama la atención que ante aspectos actitudinales y de relaciones cienciatecnología-sociedad no existen diferencias significativas entre el alumnado de diferentes modalidades.
\end{abstract}

Palabras clave: alfabetización científica; enseñanza de las ciencias

In what aspects is the scientific literacy of high school students more deficient?

Abstract: In this article, a study is carried out on various definitions of scientific literacy. Based on them, a series of indicators is developed and an experimental design is presented that aims to assess the degree of scientific literacy of the scientific and technological high school students (GCT) and that of the humanities and social sciences (GHS) in these aspects, comparing them each. The results show that conceptually the GCT students have a higher degree of scientific knowledge than the GHS students. On the other hand, it is striking that in the face of attitudinal and science-technology-society relations there are no significant differences between students of different modalities.

Keywords: Scientific literacy; science teaching

Para citar este artículo: Balastegui M., Palomar R., Solbes J. (2020) ¿En qué aspectos es más deficiente la alfabetización científica del alumnado de Bachillerato?. Revista Eureka sobre Enseñanza y Divulgación de las Ciencias 17(3), 3302. doi: 10.25267/Rev_Eureka_ensen_divulg_cienc.2020.v17.i3.3302

\section{Introducción}

El concepto de alfabetización científica, es muy polisémico, y por eso en este trabajo se realiza, en primer lugar, un análisis bibliográfico para intentar encontrar una visión global de la misma, que incluya el mayor número de objetivos relevantes para la educación científica.

$\mathrm{Si}$ bien es cierto que existen numerosos trabajos dedicados a definir el concepto de alfabetización científica, como veremos en el marco teórico, son pocos los que tratan de medirla (Laugksch y Spargo 1996; Özdem, Çavas, Çavas, Çakıroglu y Ertepınar 2010) y estos se limitan a solo algunos aspectos de la misma (conceptos, naturaleza de la ciencia NdS) y suelen utilizar ítems cerrados. Por ello, en este trabajo pretendemos utilizar un concepto amplio de alfabetización científica extraído de la investigación para diseñar un cuestionario que 
pueda evaluar el grado de alfabetización científica del alumnado de bachillerato en diferentes aspectos con cuestiones más abiertas.

Así, nos planteamos los siguientes problemas: ¿en qué medida el alumnado logra los objetivos de alfabetización científica propuestos? y chay o no diferencias al respecto entre los estudiantes del bachillerato científico tecnológico y el de humanidades y CC.SS.? Esta comparación tampoco es habitual en la bibliografía, pero pensamos que estas diferencias pueden contribuir a mostrar los efectos de la enseñanza de las ciencias en la alfabetización científica.

\section{Marco teórico}

Aunque el concepto de alfabetización científica es actual en cuanto a su importancia e interés, tiene sus orígenes a finales de los años cincuenta y se entiende como la familiaridad del público en general con la ciencia (Deboer 2000). Durante la última década del siglo XX y principios del XXI el interés por la alfabetización científica creció gracias a investigaciones didácticas, diseños de nuevos currículos y desarrollo de metodologías educativas que fomenten una alfabetización amplia y completa de los ciudadanos. Aunque Roberts (2007) señala que hay distintas visiones de la alfabetización científica, podemos encontrar convergencias entre las mismas. Así, Bybee (1997) sintetiza el concepto de alfabetización científica como una metáfora que permite enriquecer el contenido que le damos a los términos científicos. De esta forma, la alfabetización científica, aunque debe incluir el dominio del vocabulario científico, no ha de limitarse a esa definición funcional.

Atendiendo a esta alfabetización dirigida a la ciudadanía, Marco (2000) plantea tres aspectos básicos que debe incluir la alfabetización científica: práctico, cívico y cultural. El primero de ellos permite utilizar los conocimientos de la vida cotidiana, el segundo permite que las personas puedan intervenir socialmente, con criterio científico, en decisiones políticas y, el último que cuestionen el significado de la ciencia y la tecnología, así como de su incidencia en la sociedad.

Otros autores (Harlen 2002; Solbes y Vilches 2004; España y Prieto 2010) incluyen una definición más amplia de alfabetización científica dónde, además del propio conocimiento científico, sus áreas y contexto de aplicación, incluyen la importancia de los procesos mentales involucrados en el tratamiento de una pregunta o asunto. Tener una visión adecuada de los problemas a los que se enfrenta la humanidad actualmente, sus causas y posibles medidas que se puedan adoptar, favorece la toma de decisiones.

Esta descripción de alfabetización lleva asociada la importancia de la educación para su adquisición. Reid y Hodson (1993) proponen una educación dirigida hacia una cultura científica básica que, además de incluir el conocimiento de la ciencia (hechos, conceptos y teorías), proporcione aplicaciones, habilidades y tácticas en la resolución de problemas que permita la interacción con la ciencia y la tecnología a través de cuestiones socio-económicopolíticas y ético-morales. Todo ello se refuerza con un estudio de la naturaleza de la ciencia y un acercamiento a la práctica científica en la escuela. Actualiza y amplía la propuesta en Hodson (2014), donde señala que la enseñanza aprendizaje de la ciencia ha de incluir:

- Aprender ciencia, mediante la adquisición y el desarrollo de conocimiento teórico y conceptual. Esto implica usar el conocimiento científico para describir, explicar y predecir fenómenos naturales, así como para analizar problemas y adoptar decisiones en contextos personales y sociales.

- Aprender sobre ciencia, para conseguir una comprensión básica de la naturaleza de la ciencia, así como de las complejas interacciones entre ciencia, tecnología y sociedad 
(CTS). Esto conlleva comprender las características de la ciencia, valorar la calidad de una información científica a partir de la procedencia y de los procedimientos empleados para producirla y entender cómo se elaboran los modelos y las teorías, qué utilidad tienen y por qué se modifican.

- Aprender a hacer ciencia, con el desarrollo de destrezas y actitudes adecuadas para la indagación científica y la resolución de problemas. Esto supone identificar cuestiones científicas, formular hipótesis y diseñar estrategias para comprobarlas; buscar y seleccionar información relevante para el caso que se analiza; procesar información, recoger e interpretar datos cuantitativos y cualitativos, leer e interpretar gráficos, hacer correlaciones y diferenciar entre correlación y causalidad; construir una argumentación consistente o valorar la calidad de otra y alcanzar conclusiones basadas en hechos, datos, observaciones o experiencias.

- Aprender a tratar cuestiones socio-científicas (CSC), desarrollando el pensamiento crítico para participar, analizar y tomar decisiones responsables ante estos problemas. Esto implica: interesarse por CSC y hacer indagaciones; valorar la influencia social de los productos de la ciencia y la tecnología y debatir sobre cuestiones científicas y tecnológicas de interés social y responsabilizarse adoptando medidas que eviten el agotamiento de los recursos naturales o el deterioro ambiental y favorecer un desarrollo sostenible.

Ante esta visión común de una ciencia para todos como un objetivo educativo de la sociedad, hay algunos autores que consideran la cultura científica universal como un objetivo inútil (Shamos 1995). Las investigaciones de Shamos ponen en duda la necesidad de una alfabetización científica para toda la ciudadanía basándose principalmente en el modo en que enseñamos ciencia, convencido que la mayoría de estudiantes salen de las clases de ciencias con una comprensión intelectual, pero sin ninguna apreciación pragmática.

Por tal de combatir esa visión descontextualizada de la ciencia que muchos estudiantes puedan tener, se han diseñado propuestas didácticas que fomentan aspectos procedimentales y actitudinales que conectan las aulas de ciencias con la sociedad. Sanmartí (2010) muestra una perspectiva multidisciplinar de la ciencia ya que con actividades de ámbito científico se desarrollan competencias lingüísticas que permiten leer y entender artículos, y su posterior análisis. Otros trabajos también relacionan la educación científica básica con una dimensión superior denominada "enseñar a pensar" (Prieto, España y Martín 2011). En estos trabajos se ponen de manifiesto algunos problemas que presenta el alumnado, no exclusivamente relacionado con la ciencia y su naturaleza, sino con la comprensión lectora y las expresiones oral y escrita.

Los trabajos sobre alfabetización científica no van exclusivamente dirigidos a la población en general. Vilches, Solbes y Gil (2004) además de defender una alfabetización científica común para todos los ciudadanos, hacen hincapié en el poder de ésta como base en la preparación de los futuros científicos ya que en las carreras científicas universitarias pierden peso curricular aspectos que muestren las relaciones ciencia-tecnología-sociedad.

Otra vertiente de la alfabetización científica vinculada con un aspecto más personal y no tan práctico, es la relacionada con los aspectos morales y afectivos que el conocimiento científico puede ofrecer a las personas. Sandler (2004) analiza la moralidad y las emociones unidas a la toma de decisiones de los científicos, considerándolas inseparables del individuo. Así mismo, Matthews (1994) resume un conjunto de situaciones problemáticas actuales de la ciencia y la tecnología que muestran la necesidad de hacer uso de cuestiones éticas para poder resolverlas. 
La relevancia de la ciencia en la escuela está relacionada directamente con el objetivo de alfabetización científica ya que su significado responde a la pregunta ¿Por qué es relevante enseñar ciencia? La respuesta a esta interrogación es múltiple: ciencia para proseguir estudios científicos, ciencia para tomar decisiones en los asuntos públicos, ciencia funcional para trabajar en las empresas, ciencia para seducir al alumnado, ciencia útil para la vida cotidiana, ciencia para satisfacer las curiosidades personales y ciencia como cultura (Acevedo 2004).

Para poder favorecer esta alfabetización científica desde la escuela es necesario mejorar algunos aspectos, ya estudiados por la didáctica de las ciencias experimentales que ha puesto de manifiesto la falta de motivación del alumnado. Generalmente, si el alumnado no percibe la utilidad de lo que deben de aprender, el interés y el esfuerzo tiende a disminuir (Alonso y Montero 2001). Por este motivo, algunos autores han investigado sobre aspectos de la cultura científica básica que puedan despertar interés en el alumnado por el conocimiento científico. En esta línea, Solbes y Traver (2001) demuestran experimentalmente cómo la introducción de la historia y epistemología de las ciencias en las clases de física y química ayuda a promover la motivación y aumentar el interés del alumnado de ciencias. Con el mismo objetivo, otros autores (Sadler 2009; España y Prieto 2010; Zeidler 2014; Díaz Moreno 2018) defienden la utilización de CSC como contexto de aprendizaje y promover así el desarrollo de un pensamiento crítico y científico del alumnado (Torres y Solbes 2016; Gil y Vilches 2004).

Algunos trabajos van más allá de una alfabetización científica para educar y buscan un objetivo más individual y personal: la cultura científica como fuente de placer personal (Gil y Vilches 2004). Otros autores (Fensham 2002; Matthews 1994) también destacan la capacidad del estudio de las ciencias como fuente de placer, capaz de entusiasmar con su historia y desarrollo. Aparece así, un nuevo motivo para facilitar una educación científica para todos: el placer intelectual que nace cuando descubrimos nuevos conocimientos.

Finalmente, desde un punto de vista político y legislativo la alfabetización científica sigue siendo un tema de interés y en desarrollo. Los países de la OCDE reconocen que los estudiantes deben desarrollar unas habilidades y conocimientos relacionados con las prioridades del siglo XXI (Bybee y Fuchs 2006). Por ejemplo, en PISA 2006 (Bybee, McCrae y Laurie 2009) se refieren a cuatro características relativas a cada individuo interrelacionadas con la alfabetización científica: el contexto personal, social y global, que incluye las situaciones cotidianas en las que se favorece un contacto con la ciencia y tecnología; las competencias científicas, que engloban la identificación, explicación y uso de las cuestiones científicas; el conocimiento científico y las actitudes ante la ciencia.

Con esta base teórica, hemos considerado necesario elaborar unos indicadores que nos permitan evaluar el grado de alfabetización científica del alumnado una vez finalizada la Educación Secundaria Obligatoria. Para ello se han sintetizado los siguientes objetivos de dicha etapa educativa basados en el marco teórico, en el análisis de los currículums educativos y en las pruebas PISA (Moreno, Benítez y Cercadillo 2010):

- Utilizar el método científico para entender mejor los fenómenos naturales y resolver problemas que su estudio plantea.

- Utilizar conceptos de forma cualitativa para explicar situaciones que tienen lugar en nuestro entorno.

- Analizar y reconocer la importancia y repercusiones para la sociedad y el medio ambiente de la ciencia y la tecnología.

- Interpretar información científica y utilizarla para formarse una opinión propia, expresarse adecuadamente mediante juicios y tomar decisiones.

- Argumentar, redactar y debatir temas científicos utilizando correctamente la lengua. 
- Comprender la naturaleza de la ciencia.

- Desarrollar una actitud positiva hacia la ciencia y su aprendizaje.

Así, nuestros objetivos de investigación serán evaluar en qué medida el alumnado logra los objetivos de alfabetización científica propuestos anteriormente y, además, averiguar si hay o no diferencias al respecto entre los estudiantes del bachillerato científico tecnológico y el de humanidades y CC.SS.

\section{Diseño experimental}

En este apartado se presenta el diseño experimental que permite evaluar el grado de alfabetización científica del alumnado de bachillerato. Para ello se ha elaborado un cuestionario para comprobar en qué medida el alumnado logra los objetivos indicados en la introducción. Normalmente estos cuestionarios (Laugksch y Spargo 1996; Özdem et al. 2010) sólo evalúan algunos aspectos de la alfabetización científica (conceptos, naturaleza de la ciencia NdS) y suelen utilizar ítems cerrados (elección múltiple, $\mathrm{V}$ o F). Basándonos en los mismos, se han desarrollado los ítems 1 y 2. Pero para evaluar objetivos como los propuestos anteriormente a resultas del análisis bibliográfico (argumentación, indagación, contextualización, etc.), son necesarias cuestiones abiertas. Entre ellas, el ítem 8.1 proviene de las pruebas PISA (Moreno et al. 2010), el ítem 5 está basado en ideas de Huxley de "Un mundo feliz" y de Ustinov, en un documental sobre la vida de Einstein y el resto son de redacción propia. La preparación del cuestionario ha contado con las siguientes fases: análisis del borrador por un equipo de expertos y realización de un ensayo piloto que han permitido analizar la validez del cuestionario revisado y determinación de la fiabilidad mediante el test de alfa de Cronbach.

Para la elaboración de los ítems de los cuestionarios se ha relacionado previamente cada uno de ellos con los objetivos de la alfabetización científica en secundaria, como puede observarse en la Tabla 1.

Tabla 1. Relación de los objetivos de alfabetización científica con los ítems del cuestionario

\begin{tabular}{|l|l|}
\hline $\begin{array}{l}\text { 1. Utilizar el método científico para entender mejor los fenómenos naturales y resolver } \\
\text { problemas que su estudio plantea. }\end{array}$ & I, 4 \\
\hline $\begin{array}{l}\text { 2. Utilizar conceptos de forma cualitativa para explicar situaciones que tienen lugar en nuestro } \\
\text { entorno. }\end{array}$ & Ítem $1,2,7$ \\
\hline $\begin{array}{l}\text { 3. Analizar y reconocer la importancia y repercusiones para la sociedad y el medio ambiente de } \\
\text { la ciencia y la tecnología. }\end{array}$ & Ítem 6 \\
\hline $\begin{array}{l}\text { 4. Interpretar información científica y utilizarla para formarse una opinión propia, expresarse } \\
\text { adecuadamente mediante juicios y tomar decisiones. }\end{array}$ & Ítem 5, 8 \\
\hline $\begin{array}{l}\text { 5. Argumentar, redactar y debatir temas científicos utilizando correctamente el lenguaje. } \\
\text { 6. Comprender la naturaleza de la ciencia. }\end{array}$ & Ítem 5 \\
\hline 7. Desarrollar una actitud positiva hacia la ciencia y su aprendizaje. & Ítem 9,10 \\
\hline
\end{tabular}

Para poder realizar el tratamiento estadístico de los datos hemos dividido los 10 ítems del cuestionario en dos bloques: preguntas de seis puntuaciones (0-5) y preguntas de puntuación correcta-incorrecta (1-0). El primer tipo de preguntas está formado por los ítems 9.1, 9.2, 9.3, 9.4, 9.5, 10 (ver Tabla 2) y tiene un valor de alfa de Cronbach 0,834. Por otro lado, el apartado del cuestionario de puntuación 0-1 está formado por el resto de ítems (ver Tabla 2) con un valor de alfa de Cronbach de 0,718, valores que ofrecen fiabilidad al cuestionario, ya que el valor aceptado para la misma es de 0,7 en adelante. 
Tabla 2. Ítems del cuestionario de alfabetización científica para alumnado de Bachillerato
1. Señalad si consideráis verdaderas $(\mathrm{V})$ o falsas $(\mathrm{F})$ las siguientes afirmaciones:
1.1 La masa de un cuerpo es igual a su peso.
1.2 Es lo mismo calor que temperatura.
1.3 Un cuerpo frío contiene calor.
1.4 El punto de ebullición del agua es únicamente $100^{\circ} \mathrm{C}$.
1.5 La energía únicamente puede existir en los seres vivos.
1.6 Cuando la energía se transfiere de un cuerpo a otro, parte de la energía se pierde.

\section{Elegid la respuesta correcta:}

2.1 ¿Cuál es el gas más abundante en el aire?

a) Dióxido de carbonob) Nitrógeno c) Oxígeno d) Hidrógeno

2.2 ¿Qué significa la "A" en emisiones de radio en AM?
a) Amplitud b) Amperios c) Ampliación

2.3 ¿Qué elemento principalmente contienen los compuestos de Química Orgánica?

a) Hidrógeno b) Carbono c) Cloro d) Nitrógeno

2.4 ¿Qué letra se utiliza en Física para referirnos a la velocidad de la luz?

a) “a" b) "b" c) "c" d) "l"

2.5 ¿Cuál es el gas noble más pesado?

a) Xenón b) Neón c) Radón d) Helio

2.6 Aproximadamente ¿Cuál es la edad de la Tierra?

a) 7000 años b) 100000 años c) 4,5 millones de años d) 4500 millones de años

2.7 ¿Cuáles son las unidades de medida de la resistencia eléctrica?

a) Joule b) Watt c) Ohm d) Hertz

2.8 ¿Qué elemento del sistema periódico tiene el símbolo K?

Sodio b) Litio c) Potasio d) Calcio

3. Indicad mediante una hipótesis de qué depende el espacio recorrido por un coche de juguete que circula por la clase a velocidad constante. Diseñad y realizad una investigación para comprobar la hipótesis.

4. Existen diferentes teorías sobre el modelo atómico a lo largo de la historia. ¿Por qué se ha cambiado de unas a otras?

5. Presentad argumentos a favor y/o en contra de cada una de las siguientes afirmaciones.

5.1 Un científico no tiene sólo la obligación de investigar, sino que también tiene la responsabilidad ética sobre las consecuencias de aquello que produce su ingenio. Peter Ustinov

5.2 La ciencia y la técnica, al servicio de los intereses del poder, llevarán al mundo a formas sociales de dominación absoluta, a instituciones opresoras en las que nada quedará al margen, de las que nadie escapará. Aldous Huxley

6. Comentad brevemente algún aspecto destacado sobre las siguientes problemáticas, en caso de reconocerlas.

6.1 COP (Contaminantes Orgánicos Persistentes)

6.2 Uso del DDT como insecticida

6.3 Destrucción de la capa de ozono

6.4 Incremento del efecto invernadero

7. Describe brevemente situaciones cotidianas en las que utilizas conceptos científicos. (Por ejemplo: lectura de las calorías de una etiqueta de alimentos; utilizar diferentes métodos de cocción en la cocina...)

8. Leed la siguiente información y contestad a las preguntas que aparecen a continuación.

¿Qué actividades humanas contribuyen al cambio climático?

La combustión del carbón, la gasolina y el gas natural, así como de la deforestación y diversas prácticas agrícolas e industriales, alteran la composición de la atmósfera y contribuyen al cambio climático. Estas actividades humanas han originado un aumento de la concentración de particulas y gases de efecto invernadero en la atmósfera. La importancia relativa de los principales causantes del cambio de temperatura se representa en el siguiente gráfico. 


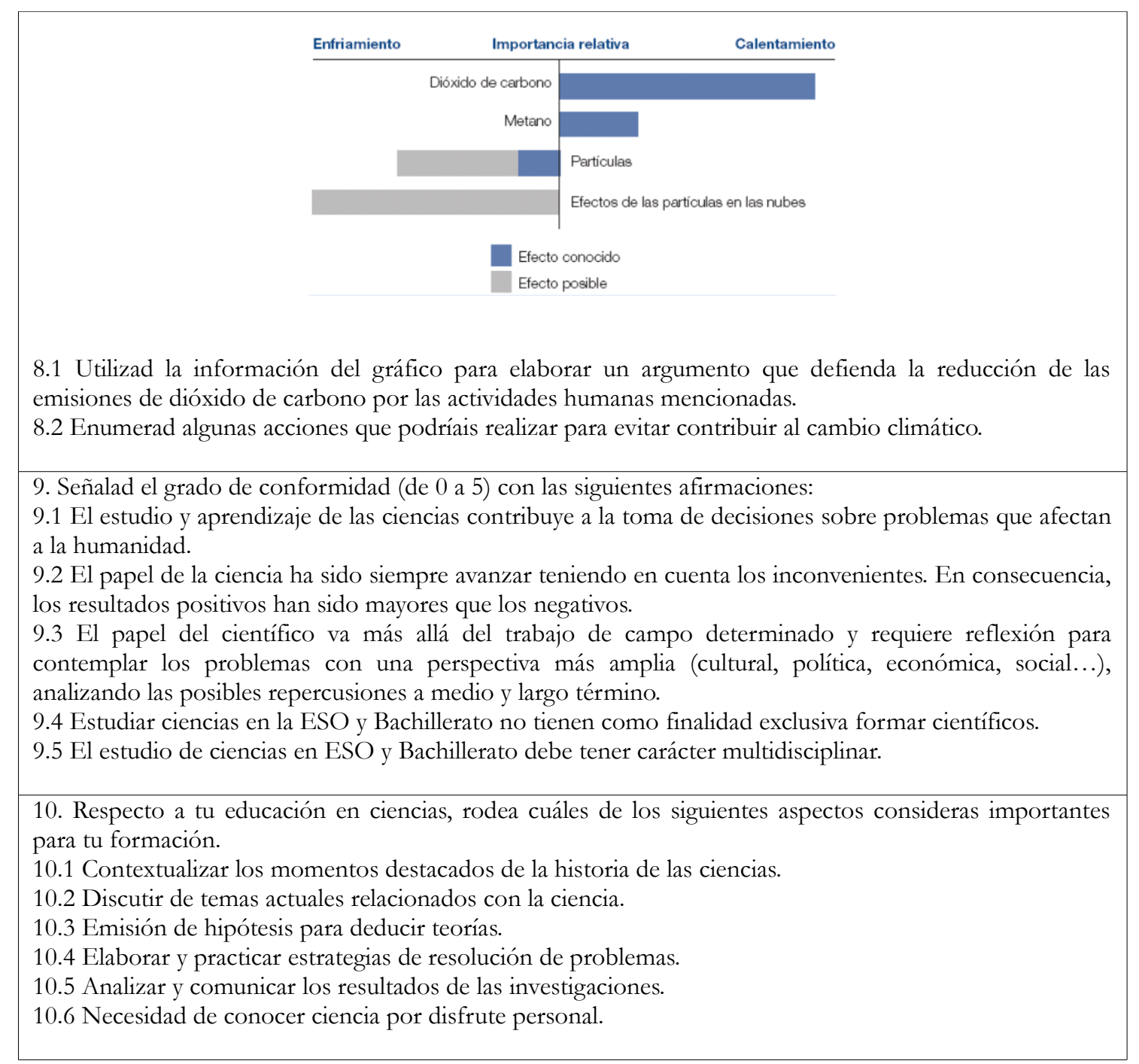

El cuestionario está dirigido al alumnado de Bachillerato tanto de la modalidad científicotecnológica como de la de humanidades y CC.SS. ya que ambos han finalizado la Educación Secundaria Obligatoria que incluye la cultura científica básica que el sistema educativo facilita a los ciudadanos.

El cuestionario se ha pasado a 104 estudiantes de primero y segundo de Bachillerato de dos centros públicos de la Comunidad Valenciana, 54 de la modalidad de Ciencia y Tecnología (GCT) y 50 de Humanidades y CCSS (GHS).

\section{Resultados}

En primer lugar, se han comparado las puntuaciones globales del cuestionario obtenidas para cada grupo, para analizar las diferencias estadísticamente significativas entre grupos en el resultado global del cuestionario. Después se analizan ítem a ítem los resultados de cada uno de los grupos.

\section{Comparación de la media de la puntuación total del cuestionario}

Con el objetivo de realizar la comparación entre grupos de las medias obtenidas en el total del cuestionario se procede a comprobar si esta variable sigue una distribución normal, en cuyo caso podremos utilizar la prueba t-Student para esta comparación. Mediante la prueba Kolmogorov-Smirnov se comprueba la normalidad de la muestra, así que se procede a calcular 
la media de las puntuaciones globales del cuestionario para cada grupo y modalidad, para comparar entre sí a través de la prueba t-Student, como muestra la Tabla 3.

Tabla 3. Puntuaciones globales del cuestionario y su comparación entre modalidades diferentes con t-Student (* indica diferencias estadísticamente significativas)

\begin{tabular}{|l|c|c|c|}
\hline Grupo & $\mathbf{N}$ & Ítems 1 a 8 & Ítems 9 y 10 \\
\hline & & Media (d.e.) & Media (d.e.) \\
\hline GCT & 54 & $15,22(0,37)$ & $17,37(0,47)$ \\
\hline GHS & 50 & $12,06(0,44)$ & $17,64(0,43)$ \\
\hline Significación asintótica bilateral & $0,00^{*}$ & 0,67 \\
\hline
\end{tabular}

Las preguntas del cuestionario con respuesta correcta-incorrecta (1-0) engloban los ítems del 1 al 8 y evalúan aspectos conceptuales, procedimentales y de relaciones CTS. Como el número total de ítems evaluables en esta parte del cuestionario es de 26 , el valor máximo de la media es 26. Los 5 subapartados del ítem 9 y el ítem 10, sobre el papel y la naturaleza de la ciencia, tienen puntuaciones de 0 a 5 , con lo que el valor máximo para la media en estos dos ítems será 30. En la Tabla 3 puede apreciarse que ningún grupo se aproxima al valor máximo de la puntuación global y que los valores de las medias están muy por debajo, aspecto que pone de manifiesto un bajo grado de alfabetización científica del alumnado.

Así mismo, se observa en los valores de la significación asintótica bilateral existe diferencia estadísticamente significativa entre los grupos GCT y GHS, como cabría esperar ya que el grupo GHS no han continuado un itinerario científico y, por tanto, sólo han cursado mayoritariamente los contenidos científicos obligatorios de la ESO y Ciencias para el mundo contemporáneo (asignatura lamentablemente eliminada en la LOMCE). Estas diferencias entre grupos, pese a ser significativas, apenas pasan del 10\%, lo que estaría indicando el poco impacto en la alfabetización científica que tiene la modalidad escogida en bachillerato. En cambio, en los ítems que evalúan aspectos actitudinales, 9 y 10, no existe diferencia estadísticamente significativa entre los grupos, lo que señala que el conocimiento sobre el papel y la naturaleza de la ciencia es similar en ambos grupos, posiblemente debido a que este sea un hecho muy poco trabajado en las asignaturas científicas (Solbes y Traver 2001).

Ante estos resultados, es necesario analizar ítem a ítem del cuestionario para ver dónde se encuentran las diferencias o similitudes en las respuestas del alumnado.

\section{Comparación de los resultados por los objetivos del cuestionario}

En este apartado se compararán los resultados de la puntuación total de cada ítem de los grupos GCT y GHS ya que los resultados han puesto de manifiesto que sí que existe una diferencia estadísticamente significativa en las cuestiones más conceptuales y procedimentales, pero no en las actitudinales (Ver Tabla 3). 


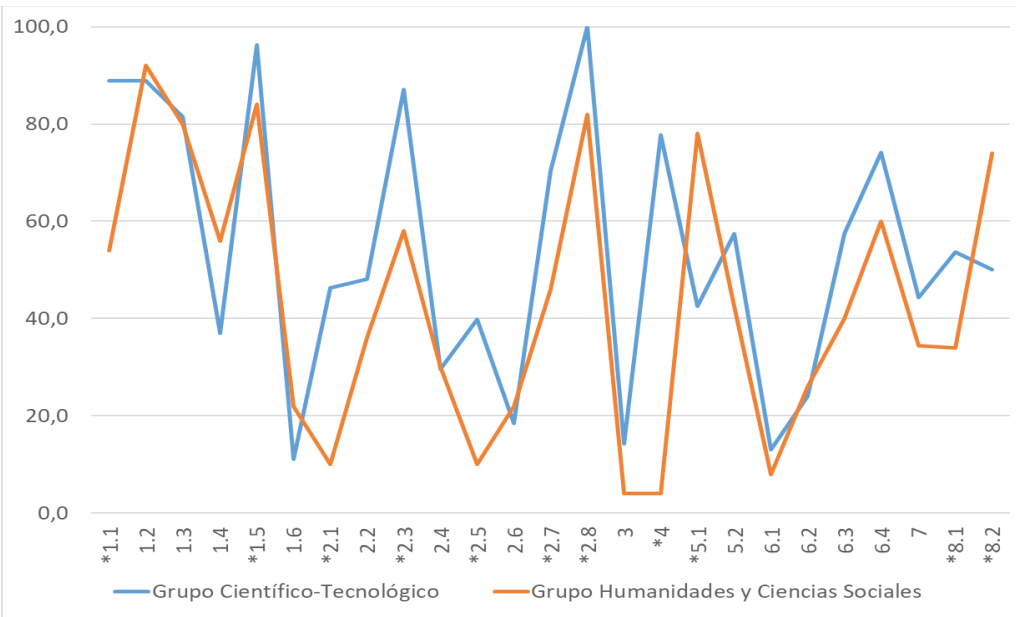

Figura 1. Porcentaje de respuestas correctas y comparación entre grupos del cuestionario ítems 1 a 8 (* indica diferencias estadísticamente significativas)

Como puede observarse en la Figura 1 existen algunas diferencias entre los dos grupos en las respuestas a cada uno de los ítems. Con el objetivo de ver si estas diferencias son estadísticamente significativas, y dado que se trata de comparar frecuencias de variables categóricas en dos grupos, se escoge la prueba de Chi-cuadrado con valor de s.a.b. menor que p-value 0,05. Aparecen diferencias estadísticamente significativas entre ambos grupos, y se observa como el grupo de ciencias destaca sobre el de humanidades y CC.SS. en las cuestiones conceptuales (en concreto, en los ítems 1.1, 1.5, 2.1, 2.3, 2.5, 2.7 y 2.8). Este resultado es esperado ya que el alumnado de ciencias continúa su formación en el ámbito que exploran los ítems mientras que el grupo de humanidades y CC.SS. puede que desde $3^{\circ}$ de ESO no haya trabajado ninguna materia científica, a la que debemos añadir la extinta Ciencias para el mundo contemporáneo. De igual modo sucede en el ítem 4 en el que el alumnado del GCT es capaz de explicar por qué se cambia de un modelo a otro, y en cambio en el GHS hay pocas respuestas correctas.

En cambio, a nivel de $\mathrm{NdC}$ y procedimientos (ítem 3), no existe diferencia estadísticamente significativa. En este caso el alumnado debía formular una hipótesis y realizar un diseño experimental que la pusiese a prueba. Aunque el porcentaje de respuestas correctas es ligeramente superior en el grupo de ciencias, la mayoría de ellos no son capaces de plantear una hipótesis y describir algún diseño que la ponga a prueba. Esto puede ser debido a que gran parte alumnado no entrena estas destrezas, y puede haber pasado toda la educación secundaria sin formular hipótesis ni realizar experimentos en el laboratorio (Solbes, Montserrat y Furió, 2007).

A nivel de estrategias argumentativas (ítem 5.1) los resultados ponen de manifiesto que las del alumnado del grupo GHS son mejores, tienen una visión más crítica de la actividad científica y defienden una responsabilidad ética por parte de los científicos. Así, las respuestas del grupo GCT se caracterizan por una ausencia de responsabilidad ética en la actividad científica. Ven como objetiva la tarea del investigador y creen que una tercera persona (la que utiliza la investigación) es la responsable de las consecuencias. También aparece la visión de una ciencia que muestra la verdad, invariable y en busca de mejoras para los humanos, actitudes bastante cientifistas:

- Alumno 1. Un cientifico puede investigar sobre cualquier cosa, el problema está cuando otras personas tratan de dar un mal uso a estas. 
- Alumna 1. Pienso que un cientifico debe dedicarse únicamente a investigar para mejorar las cualidades de vida, no debería preocuparse de la ética para que ésta depende de cada persona, a cada uno le parecerá bien o mal el avance cientifico.

- Alumno 4. Un científico debe buscar la verdad útil para la bumanidad y presentarla, pero no tiene por qué responsabilizarse del que otro haga con ese conocimiento.

Las respuestas del grupo GHS se caracterizan por un mayor apoyo a la responsabilidad del investigador. Estos muestran que la ciencia debe avanzar, independientemente de lo que la sociedad acepte, pero teniendo en cuenta no afectar el medio que le rodea. Sin embargo, consideran que a la hora de investigar aparecen aspectos negativos y positivos, pero que la responsabilidad del científico debe hacer pública aquellas acciones que puedan ser perjudiciales. Al contrario que sus compañeros de ciencias manifiestan pensamiento crítico respecto a la actividad científica:

- Alumna 3. Estoy a favor, ya que tendría que ver las consecuencias de lo que investiga, si podría afectar negativamente a la sociedad o al medio ambiente (en conjunto) debería pensar en hacerlo o no.

- Alumno 5. Estoy completamente a favor ya que lo producido por él debe recaer sobre su conciencia. Si estás en contra de las bombas atómicas, no investigas en su desarrollo, y al igual en todos sus ámbitos.

- Alumno 6. Totalmente a favor, porque a la hora de investigar, dentro de la propia palabra tiene implicito investigar consecuencias tanto positivas como negativas que va a tener.

Las respuestas del ítem 5.2 muestran resultados similares entre ambos grupos. Algunos consideran la afirmación Huxley como una situación ficticia y con pocas posibilidades de que pueda llegar a ocurrir. Pero también existen aquellas argumentaciones que consideran esta situación como posible o como real en la actualidad, poniendo como ejemplo principal los conflictos bélicos. En GCT encontramos respuestas como:

- Alumno 2. Estoy de acuerdo. Un ejemplo son las guerras donde se utilizan armas químicas, un país gana la guerra y ha sometido al perdedor.

- Alumno 4. Una de las cosas malas de la ciencia y la manipulación cientifica es que pueden llegar a ser muy peligrosas, y si acaban en manos de personas que sólo miran por sus intereses y no en las consecuencias que puede llegar a tener, puede llegar a ser una catástrofe.

En GHS encontramos respuestas semejantes:

- Alumna 2. El aprovechamiento de los avances cientificos, mayoritariamente por organizaciones, empresas o gobiernos, dará lugar a una más marcada diferenciación de poderes y de niveles de vida entre grupos sociales. hará más marcada la desigualdad. Además, el grupo de personas (probablemente una mayoría) que no disponga de estos avances estarian bajo de la voluntad de los poderosos, sin posibilidad de salir de este sistema.

- Alumna 3. Estoy de acuerdo porque la ciencia es muy poderosa y amplia. Si está al servicio del poder se moverá por los intereses políticos, como en la segunda guerra mundial con la bomba atómica.

Sucede lo mismo ante el conocimiento de problemáticas ambientales (ítems 6), o existe diferencia estadísticamente significativa entre el grupo GCT y el grupo GHS, dado que el valor es mayor que 0,05. Los resultados del alumnado son similares independientemente de la modalidad de bachillerato que cursan, posiblemente por su presencia en los medios de comunicación. Esto puede estar indicándonos que la transversalidad con las que aparecen algunos de estos contenidos en el currículo, no es suficiente para que el alumnado forme una opinión duradera en el tiempo. 
Ante situaciones cotidianas relacionadas con la ciencia (ítem 7) tampoco existe diferencia estadísticamente significativa entre ambos grupos. Este resultado pone de manifiesto que el alumnado de ciencias no es capaz de nombrar un mayor número que el del ámbito humanístico. El alumnado relaciona principalmente las situaciones cotidianas con las disciplinas de física, química y biología, y en menor medida las matemáticas y la informática. Este último aspecto llama la atención ya que actualmente la tecnología y la informática forman parte fundamental del día a día del alumnado. La disciplina de química es superior al resto y significativamente superior en GCT $(n=46)$ que en GHS $(n=26)$, aspecto que muestra que el alumnado detecta numerosas situaciones cotidianas relacionadas con esta materia. Las situaciones más nombradas son alimentación, cocina, productos de limpieza, informática y estudios.

En cuanto a interpretar información científica y utilizarla para tomar decisiones (ítem 8), en 8.1 hay diferencias porque el alumnado de GCT ofrece mayor porcentaje de respuestas correctas. En cambio, en el ítem 8.2 la diferencia estadísticamente significativa se debe a unos mejores resultados en el grupo GHS. Así mismo, llama la atención que en el ítem 8.2 el GHS es capaz de nombrar un mayor número de contribuciones personales para no contribuir al cambio climático. Las más nombradas por ambos grupos son reciclaje, uso del transporte público y evitar el uso del coche privado. Otras que se mencionan, aunque en menor grado son uso de energías renovables, cuidado de zonas verdes e incendios, no contaminar y reducir el consumo de agua.

Por tanto, aunque en aspectos conceptuales el alumnado de ciencias muestra mejores resultados, en los procedimentales ya no existe tanta diferencia y en los relacionados con la sociedad incluso el alumnado de humanidades y CC.SS. muestran mejores resultados.

Veamos lo que ha sucedido en los ítems más actitudinales, comenzando con los porcentajes de respuestas a cada categoría del ítem 9.

Tabla 4. Porcentaje de respuestas en cada categoría de la escala para el ítem 9 separados por subgrupos de modalidad (* indica diferencias estadísticamente significativas)

\begin{tabular}{|c|c|c|c|c|c|c|c|c|}
\hline Ítem & Grupo & $\mathbf{0}$ & $\mathbf{1}$ & $\mathbf{2}$ & $\mathbf{3}$ & $\mathbf{4}$ & $\mathbf{5}$ & S.a.b. \\
\hline $\mathbf{9 . 1}$ & GCT & 0,0 & 0,0 & 11,1 & 22,2 & 35,5 & 31,5 & 0,40 \\
\hline & GHS & 0,0 & 2,0 & 6,0 & 34,0 & 36,0 & 22,0 & \\
\hline $\mathbf{9 . 2}$ & GCT & 14,8 & 37,0 & 20,4 & 13,0 & 11,1 & 3,7 & 0,07 \\
\hline & GHS & 6,0 & 16,0 & 38,0 & 20,0 & 14,0 & 6,0 & \\
\hline $\mathbf{9 . 3}$ & GCT & 0,0 & 3,7 & 3,7 & 13,0 & 31,5 & 48,1 & 0,25 \\
\hline & GHS & 4,0 & 4,0 & 0,0 & 24,0 & 32,0 & 36,1 & \\
\hline $\mathbf{9 . 4}$ & GCT & 20,4 & 13,0 & 20,4 & 16,7 & 22,2 & 7,4 & 0,58 \\
\hline & GHS & 12,0 & 24,0 & 22,0 & 20,0 & 18,0 & 4,0 & \\
\hline $\mathbf{9 . 5}$ & GCT & 1,9 & 3,7 & 5,6 & 27,8 & 25,9 & 35,2 & 0,64 \\
\hline & GHS & 2,0 & 2,0 & 12,0 & 32,0 & 30,0 & 22,0 & \\
\hline
\end{tabular}

En la Tabla 4 se puede observar que no existe ninguna diferencia estadísticamente significativa (prueba Chi-cuadrado con valor de s.a.b. menor que p-value 0,05 ) entre ambos grupos en la actitud que presentan ante la actividad científica y el estudio y aprendizaje de las ciencias. Como ponen de manifiesto algunas investigaciones (Rocard et al. 2007; Solbes et al. 2007; Vázquez y Manassero 2008) la actitud ante las ciencias es bastante negativa, pero es de destacar que no haya diferencia entre un alumnado que ha decidido cursar un itinerario científico y el que no.

Respecto a los aspectos que consideran importantes para su formación (ítem 10) otorgamos la puntuación de 0 a 5 según el número de afirmaciones que haya señalado, siendo la mínima puntuación si señala una o ninguna, y 5 la máxima si señala las 6. 
Tabla 5. Porcentaje de respuestas en cada categoría de la escala para el ítem 10 separados por subgrupos (* indica diferencias estadísticamente significativas)

\begin{tabular}{|c|c|c|c|c|c|c|c|}
\hline Ítem & Grupo & $\mathbf{0}$ & $\mathbf{1}$ & $\mathbf{2}$ & $\mathbf{3}$ & $\mathbf{4}$ & $\mathbf{5}$ \\
\hline $\mathbf{1 0}$ & GCT & 44,4 & 53,7 & 37,0 & 37,0 & 27,8 & 38,9 \\
\hline & GHS & 62,2 & 70,0 & 44,0 & 44,0 & 34,0 & 54,0 \\
\hline & S.a.b. & 0,08 & 0,11 & 0,55 & 0,55 & 0,53 & 0,17 \\
\hline
\end{tabular}

Como se puede observar en la Tabla 5 no existe diferencia estadísticamente significativa entre las respuestas de ambos grupos. En esta pregunta la mayoría del alumnado de ambos grupos ha elegido sólo dos opciones, donde la más señalada es la segunda opción, discutir de temas actuales relacionados con la ciencia. Esta opción muestra cómo el alumnado demanda ser partícipe de la construcción del conocimiento en las clases de ciencia, de forma activa, como por ejemplo realizando actividades de tipo debate. Otra afirmación que señala el alumnado en gran medida es la primera, contextualizar los momentos destacados en la historia de las ciencias. Esta es una de las carencias que generalmente se encuentra en las aulas de ciencia tradicionales y que no favorecen la visión amplia y contextualizada del conocimiento científico (Solbes y Traver 2001). En general, el alumnado demanda conectar el conocimiento científico que trabajan en las aulas con su día a día.

\section{Conclusiones}

En nuestro intento por evaluar la alfabetización científica del alumnado que ha terminado la educación secundaria y se encuentra cursando bachillerato, hemos encontrado que, de manera general, el alumnado de bachillerato no está alfabetizado en el ámbito científico, pues obtiene puntuaciones globales bajas en el cuestionario diseñado a tal efecto y, además, no reconocen la importancia de la educación científica para el ámbito cotidiano, en coincidencia con un trabajo muy reciente que, además de los habituales conceptos y $\mathrm{NdC}$, incluye las relaciones CTS en la alfabetización científica (Vizzotto y Mackedanz 2020), aunque no otros aspectos que hemos analizado en este trabajo.

Se constata también que existen diferencias según sea la modalidad de bachillerato escogida por el alumnado, en la parte del cuestionario, la más conceptual (ítems 1, 2 y 4). Esta comparación entre modalidad científico-tecnológica y de humanidades y ciencias sociales, ha demostrado que, conceptualmente, como cabía esperar, el alumnado de ciencias obtiene mejores resultados.

En el ítem 3, en que el alumnado debía formular una hipótesis y realizar un diseño experimental que la pusiese a prueba, no existe diferencia significativa, lo que lamentablemente pone de manifiesto que un gran porcentaje del alumnado de ciencias no practica estos procedimientos (Solbes et al. 2007).

Sin embargo, el alumnado de humanidades y ciencias sociales obtiene mejores resultados que el de científico-tecnológico cuando se le pide que elabore argumentaciones, aporte críticas y reflexione ante la actividad científica (ítem 5).

Ante el conocimiento de problemáticas ambientales (ítem 6) y ante situaciones cotidianas relacionadas con la ciencia (ítem 7) no hay diferencias entre ambos grupos. En el ítem 8 si las hay porque el grupo GHS nombra más contribuciones personales para no contribuir al cambio climático.

En la segunda parte del cuestionario, la que trabaja aspectos relacionados con el papel y la naturaleza de la ciencia, más actitudinales (ítems 9 a 10 del cuestionario), no se observan 
diferencias estadísticamente significativas. Así, podemos destacar de esta investigación que la actitud, no muy positiva, ante la ciencia y su estudio (Vázquez y Manassero 2008) es igual en ambos grupos: el alumnado de ciencias no tiene una mejor visión de la actividad científica que sus compañeros de humanidades.

En resumen, la comparación pone de manifiesto que el mayor nivel de enseñanza de las ciencias del alumnado del bachillerato de ciencias y tecnología respecto a los de humanidades y ciencias sociales solo contribuye a mejorar la alfabetización científica a nivel de conceptos, pero que no hay diferencias a nivel de procedimientos científicos, de $\mathrm{NdS}$, de relaciones CTS ni de actitudes hacia la ciencia. Incluso a nivel de argumentaciones sobre la actividad científica el alumnado de GHS obtiene mejores resultados que el de GCT.

Dado el bajo nivel de alfabetización científica detectado resulta incomprensible que en la LOMCE se haya suprimido la asignatura de "Ciencias para el mundo contemporáneo", cuya inclusión en ulteriores leyes educativas debería ser reivindicada por el profesorado de ciencias.

Esto nos plantea la perspectiva de investigación de realizar una serie de actividades que favorezcan la alfabetización científica con el alumnado de secundaria obligatoria, que es donde debe empezar dicha alfabetización, y evaluar la efectividad de las mismas para lograrla, que uno de los autores está realizando actualmente.

\section{Referencias}

Acevedo, J. (2004). Reflexiones sobre finalidades de la enseñanza de las ciencias: Educación científica para la ciudadanía. Revista Eureka sobre Enseñanza y Divulgación de las Ciencias, 1 (1), 3-15.

Alonso, J., Montero, I. (2001). Orientación motivacional y estrategias motivadoras en el aprendizaje escolar. Desarrollo Psicológico y Educación, 2, 259-283.

Bybee, R. (1997). Towards an Understanding of Scientific Literacy. Scientific Literacy. Kiel: LiebnizInstitut für die Pädagogik der Naturwissenschaften und Mathematik.

Bybee, R., Fuchs, B. (2006). Preparing the 21st century workforce: A new reform in science and technology education. Journal of Research in Science Teaching, 43 (4), 349-352.

Deboer, G. (2000). Scientific literacy: another look at its historical and contemporary meanings and its relationship to science education reform. Journal of Research in Teaching, . 37 (6), 582-601

Díaz Moreno, N. (2018). Caracterizando controversias sociocientíficas en la prensa escrita. Una herramienta para el desarrollo de la alfabetización científica. Revista Eureka sobre Enseñanza y Divulgación de las Ciencias, 16 (1), 1102.

España, E., Prieto, T. (2010). Problemas socio-científicos y enseñanza-prendizaje de las ciencias. Investigación en la escuela, 71, 17-24.

Fensham, P.J. (2002). Time to change drivers for scientific literacy. Canadian Journal of Science, Mathematics and Technology Education, 2, 9-24.

Gil, D., Vilches, A. (2004). La contribución de la ciencia a la cultura ciudadana. Cultura y Educación, 16 (3), 259-272.

Harlen, W. (2002). Evaluar la alfabetización científica en el programa de la OECD para la evaluación internacional de estudiantes (PISA). Enseñanza de las Ciencias, 20 (2), 209216. 
Hodson, D. (2014). Learning science, learning about science, doing science: Different goals demand different learning methods. International Journal of Science Education, 36 (15), 2534-2553.

Laugksch, R. y Spargo, P. (1996). Development of a pool of scientific literacy test-items based on selected AAAS literacy goals. Science Education, 80 (2), 121-143.

Marco, B. (2000). La alfabetización científica. En Perales, F. y Cañal, P. (coord.) Didáctica de las Ciencias Experimentales, 141-164. Alcoy: Marfil.

Mathews, M. (1994). Historia, filosofía y enseñanza de las ciencias: la aproximación actual. Enseñanza de las ciencias, 12 (1), 255-277.

Moreno, M. J.; Benítez, D.; Cercadillo, L. (comp.) (2010). Ciencias en PIS A. Pruebas liberadas. Madrid: Ministerio de Educación.

Özdem, Y., Çavas, P., Çavas, B., Çakıroglu, J. y Ertepınar, H. (2010). An investigation of elementary student's Scientific Literacy levels. Journal of Baltic Science Education, 9 (1), 619.

Prieto, T., España, E., Martín, C. (2011). Algunas cuestiones relevantes en la enseñanza de las ciencias desde una perspectiva Ciencia-Tecnología-Sociedad. Revista Eureka sobre Enseñanza y Divulgación de las Ciencias. 9 (1), 71-77.

Roberts D. A. (2007) Scientific Literacy/Science Literacy. En Abell, S. K. y Lederman, N. G. (Eds.), Handbook research on science education. Mahwah, New Jersey: Lawrence Erlbaum.

Rocard, M.; Csermely, P.; Jorde, D.; Lenzen, D.; Walberg-Henriksson, H. y V. Hemmo (2007). Science education Now: A renewed Pedagogy for the future of Europe. European Communities: Belgium.

Reid, D., Hodson, D. (1993). Ciencia para todos en secundaria. Madrid: Narcea.

Sadler, T. (2004). Moral sensitivity and its contribution to the resolution of socio-scientific issues. Journal of Moral Education, 33 (3), 339-358.

Sanmartí, N. (2010). Leer para aprender ciencias. Ministerio de Educación. Gobierno de España.

Shamos, M. (1995). The Myth of Scientific Literacy. Ner Brunswick, Rutergers University Press. New Brunswick, New Jersey.

Solbes, J.; Montserrat, R. y C. Furió (2007). El desinterés del alumnado hacia el aprendizaje de la ciencia: implicaciones en su enseñanza. Didáctica de las Ciencias Experimentales y Sociales, 21, 91-117.

Solbes, J., Traver, M. (2001). Resultados obtenidos introduciendo historia de la ciencia en las clases de física y química: mejora de la imagen de la ciencia y desarrollo de actitudes positivas. Enseñanza de las ciencias, 19 (1), 151-162.

Solbes, J., Vilches, A. (2004). Papel de las relaciones entre ciencia, tecnología, sociedad y ambiente en la formación ciudadana. Enseñanza de las Ciencias, 14 (1), 103-112.

Torres, N.Y.; Solbes, J. (2016). Contribuciones de una intervención didáctica usando cuestiones sociocientíficas para desarrollar el pensamiento crítico. Enseñanza de las ciencias. 34 (2), 43-65.

Vázquez, A.; M. A. Manassero (2008). El declive de las actitudes hacia la ciencia de los estudiantes: un indicador inquietante para la educación científica. Revista Eureka sobre Enseñanza y Divulgación de las Ciencias, 5 (3), 274-292. 
Vilches, A., Solbes, J., Gil, D. (2004). ¿Alfabetización científica para todos contra ciencia para futuros científicos? Alambique, 41, 89-99.

Vizzotto, P.A. y Mackedanz, L.F. (2020). Alfabetização Científica e a Contextualização do conhecimento: um estudo da Física aplicada ao trânsito. Revista Brasileira de Ensino de Física, 42, e20190027.

Zeidler D. L. (2014) Socioscientific Issues as a Curriculum Emphasis: Theory, Research and Practice. En Lederman, N.G y Abell, S.K. (Eds.) Handbook of Research on Science Education, II, Routledge, 697-726. 